\title{
TRANSPORT LAYER MULTIHOMING FOR FAULT TOLERANCE IN FCS NETWORKS*
}

\author{
Armando L. Caro Jr., Paul D. Amer \\ Protocol Engineering Lab \\ Computer and Information Sciences \\ University of Delaware \\ \{acaro, amer\}@cis.udel.edu
}

\author{
Randall R. Stewart \\ Cisco Systems Inc. \\ rrs@cisco.com
}

\begin{abstract}
We document a potential flaw in the current SCTP retransmission policy. The current scheme intends to improve the chance of success by exploiting the redundant paths between multihomed endpoints, but we have found that the current SCTP retransmission policy often degrades performance. We comparatively evaluate an alternative retransmission policy and show that the current SCTP retransmission policy unexpectedly performs worse under certain conditions. Our analysis exposes the problem and we discuss three possible solutions.
\end{abstract}

\section{INTRODUCTION}

Mission critical systems rely on redundancy at multiple levels to provide uninterrupted service during resource failures. Such systems when connected to IP networks often deliver network redundancy by multihoming their hosts. A host is multihomed if it can be addressed by multiple IP addresses [3]. Redundancy at the network layer allows a host to be accessible even if one of its IP addresses becomes unreachable; packets can be rerouted to one of its alternate IP addresses.

TCP does not support multihoming between two endpoints. Any time either endpoint's IP address becomes inaccessible, perhaps due to interface failure, radio channel interference, or moving out of range, TCP's connection will timeout and abort, thus forcing the application to recover. This recovery overhead and associated delay can be unacceptable for mission critical

${ }^{*}$ Prepared through collaborative participation in the Communications and Networks Consortium sponsored by the U.S. Army Research Laboratory under the Collaborative Technology Alliance Program, Cooperative Agreement DAAD19-01-2-0011. The U.S. Government is authorized to reproduce and distribute reprints for Government purposes notwithstanding any copyright notation thereon. applications in military battlefield communications where responsiveness is crucial.

To address TCP's shortcoming, the Stream Control Transmission Protocol (SCTP) has been designed with fault tolerance in mind. SCTP is an IETF (Internet Engineering Task Force) standards track transport layer protocol. Telephony signaling applications originally motivated SCTP's development, but its design makes it suitable as a general purpose transport protocol and an alternative to TCP. SCTP is a reliable, messageoriented data transport protocol that provides resistance to SYN flooding attacks, supports multiple streams to prevent head-of-line blocking, and supports multihoming for fault tolerance.

Transport layer multihoming provides the network level fault tolerance which is crucial for survivability and persistent on-the-move sessions in FCS (Future Combat Systems) networks. SCTP multihoming allow connections, or associations in SCTP terminology, to remain alive even when an endpoint's IP address becomes unreachable. SCTP has a built-in failure detection and recovery system, known as failover, which allows associations to dynamically send traffic to an alternate peer IP address when needed. Higher layer applications are unaware of the destination IP address change, as should be expected in a truly fault tolerant system.

Currently, SCTP uses multihoming for redundancy purposes only and not for load balancing. Each endpoint chooses a single destination address as the primary destination address, which is used for all data during normal transmission. Retransmitted data use alternate peer IP address(es). RFC2960 states in Section 6.4 "when its peer is multi-homed, an endpoint SHOULD try to retransmit [data] to an active destination transport address that is different from the last destination address to which the [data] was sent." 
SCTP's current retransmission policy attempts to improve the chance of success by sending all retransmissions to an alternate destination address [9]. The underlying assumption is that loss indicates either that the destination address used is unreachable, or its network path is congested. However, in wireless networks, such as in FCS networks, noisy channels significantly contribute to loss. In this case, retransmitting to an alternate destination may not increase the chance of success.

Battlefield applications are likely to experience high loss rates and require many retransmissions. Hence, while of less importance to the Internet in general, the performance of retransmissions is an important issue for persistent on-the-move sessions in FCS networks.

Regardless of the reason for loss, we have found that SCTP's current retransmission policy may actually degrade performance - even in the case of congestion induced loss. This paper documents the potential flaw in the current SCTP retransmission policy and evaluates an alternative policy. We simulated data transfers between multihomed hosts under varying loss rates. We compare transfers using the current SCTP that retransmits to an alternate destination versus a modified SCTP that retransmits to the same destination. Initial results show the modified SCTP generally provides improved performance. Under certain conditions, however, the current retransmission policy performs better. Further research is needed to improve the retransmission mechanism in general, and for FCS networks in particular.

We begin in Section 2 by describing the simulation environment used to gather data. Section 3 presents the results and analysis. We discuss some possible solutions in Section 4 and conclude the paper in Section 5.

\section{METHODOLOGY}

With support from the CTA (Collaborative Technology Alliance) Program, the Protocol Engineering Lab (PEL) at the University of Delaware (UD) implemented an SCTP module for the ns-2 network simulator $[2,4]$. This software is being used by over 50 researchers for simulating SCTP behavior. At $\mathrm{UD}$, we investigated the performance of data transfers between multihomed hosts under varying loss rates.

Figure F1 illustrates the network topology used in our simulations. We use a dual-dumbbell topology whose core links have a bandwidth of 10Mbps and a one-way propagation delay of $25 \mathrm{~ms}$. Each router is attached to five edge nodes. One of which is a dual-homed node for an SCTP agent, while the remaining four nodes are single homed nodes whose function is to introduce cross traffic that creates loss for the association. The links to the dual-homed nodes have a bandwidth of $100 \mathrm{Mbps}$ and a one-way propagation delay of $10 \mathrm{~ms}$. The single homed nodes also have 100Mbps links, but their propagation delays are randomly chosen from a uniform distribution between $5 \mathrm{~ms}$ and $20 \mathrm{~ms}$. The end-to-end one-way propagation delays range between $35 \mathrm{~ms}$ and $65 \mathrm{~ms}$. These delays roughly approximate reasonable Internet delays for distances such as coast-to-coast of the continental US and eastern US to/from western Europe. Also, each link (both edge and core links) has a buffer size equal to double the link's bandwidth-delay product. This provision is a general "rule of thumb" used by ISPs.

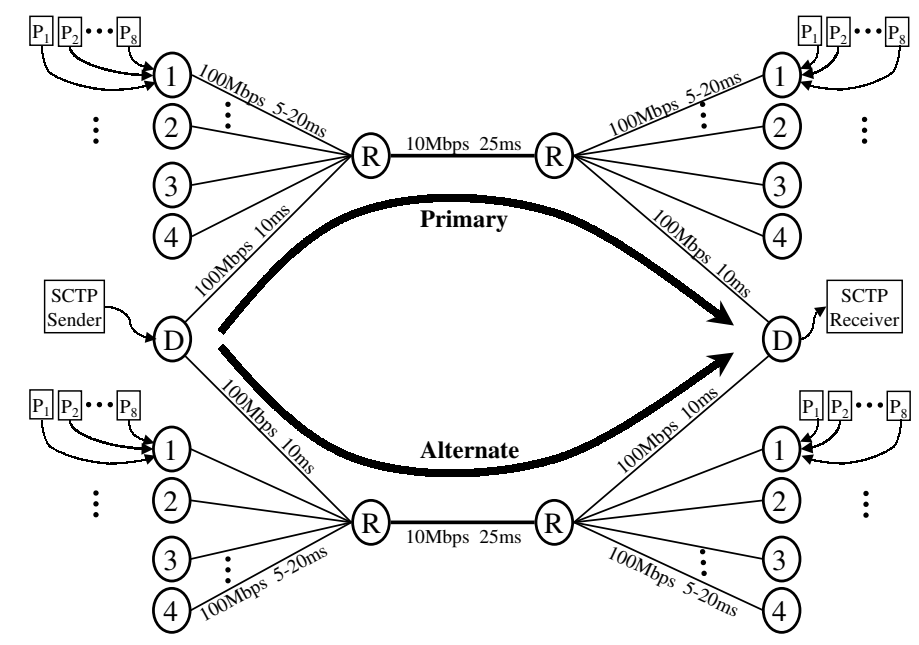

Figure F1: Simulation network topology

Our configuration has two SCTP agents (one sender and one receiver) on either side of the network, which are attached to the dual-homed edge nodes. Thus, the SCTP sender has two paths, labeled path 0 and path 1, to the SCTP receiver. Each of the single homed edge nodes has eight agents that introduce random cross-traffic according to a Pareto distribution. The cross-traffic packet sizes are chosen to closely resemble the distribution found on the Internet [1,5]. The distribution we use is as follows: $50 \%$ are $44 \mathrm{~B}, 25 \%$ are $576 \mathrm{~B}$, and $25 \%$ are 1500B. The result is an SCTP data transfer over a network with self-similar cross-traffic, which is the nature of traffic on any network [6].

We simulate a 4MB file transfer with different network conditions, which are controlled by varying the load introduced by cross-traffic. Hence, all loss experienced is due to congestion only. The aggregate levels of cross-traffic range from $5 \mathrm{Mbps}$ to $11 \mathrm{Mbps}$. Although we independently control the levels of 
cross-traffic on each of the core links, the cross-traffic on the forward and return paths are always set the same. ${ }^{1}$

Each simulation has three parameters:

1. level of cross-traffic (in Mbps) on the primary path

2. level of cross-traffic (in Mbps) on the alternate path

3. retransmission policy: current SCTP (retransmit on alternate path) versus proposed SCTP (retransmit on same path)

For each combination, we ran 60 simulations with different seeds for the random number generator.

\section{RESULTS AND ANALYSIS}

Our results compare the transfer times using two different retransmission policies under various loss rates. The first retransmission policy, labeled "current", is SCTP's current scheme of sending all retransmissions on a different path than used previously. The other policy, labeled "proposed", simply sends all retransmissions to the same destination used for the original transmission. The loss rate is calculated as the number of SCTP packets dropped divided by the number of SCTP packets transmitted.

Figure F2 presents the results for runs with a $3 \%$ loss rate on path 0 . The graph compares the file transfer time using the "current" versus "proposed" SCTP at various loss rates on path 1. Transfers using the "proposed" SCTP never use path 1 and therefore are unaffected by path 1's loss rate. These transfer times are represented as a band across all path 1 loss rates. This band outlines the upper and lower bounds of the $90 \%$ confidence interval. That is, we are $90 \%$ confident that the average transfer time lies between 34.3 and 35.1 seconds.

The completion times for transfers using the "current" SCTP which retransmit on alternate path 1 are grouped by ranges of path 1 loss rates. The graph depicts the mean and the $90 \%$ confidence interval for each of these groups. The $90 \%$ confidence interval was calculated using an acceptable error of $10 \%$. For example, the value 0.02 on the $x$-axis indicates that when the alternate path 1 has between 1.5 and $2.5 \%$ loss, the time to transfer a $4 \mathrm{MB}$ file is on average about 42.8 seconds with a $90 \%$ confidence interval between 41.1 and 44.5 seconds. As the graph shows, the "proposed" SCTP performs better for all path 1 loss rates except $0 \%$. When the path 1 loss rate is $0 \%$, both retransmission policies perform similarly.

\footnotetext{
${ }^{1}$ Due to the randomness of cross-traffic, they are never actually equal.
}

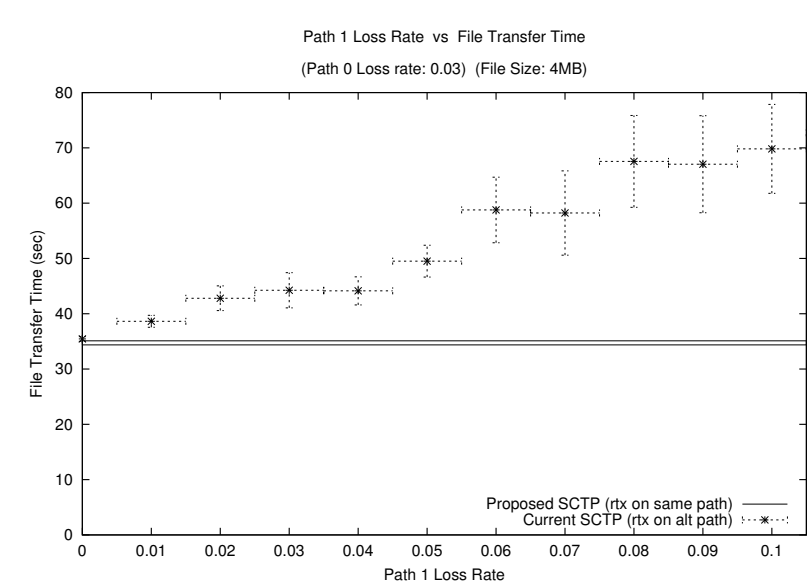

Figure F2: 4MB file transfer with $3 \%$ loss rate on path 0

Figure F3 shows results for transfers when the loss rate on path 0 is $8 \%$. When the loss rate on path 1 is less than about 4$5 \%$, the "current" SCTP retransmission policy performs better. At higher loss rates on path 1, the "proposed" SCTP yields superior performance.

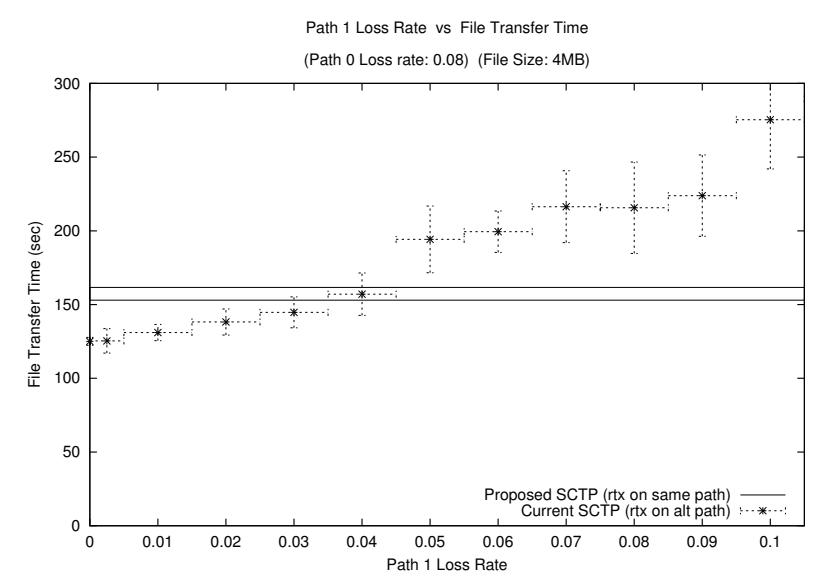

Figure F3: 4MB file transfer with $8 \%$ loss rate on path 0

We collected results for loss rates for $0-10 \%$. Due to space constraints, we could not include all graphs, but the trend remains the same. For every path 0 loss rate, the "proposed" SCTP begins performing better at some threshold. We expected the threshold to be when path 0's loss rate becomes greater than the loss rate on path 1 . It is interesting that even when the loss rate on alternate path 1 is less than on original path 0 , the file transfer often takes more time when retransmissions are sent on an alternate path. This unexpected behavior is seen in Figures F2 and F3. For example, in Figure F3 at $8 \%$ loss on path 0 and $5 \%$ loss on path 1 , it is faster to use the "proposed" scheme of only using path 0 . This behavior is not what the SCTP authors expected when specifying the current retransmission policy. 
Intuition tells us that when the loss conditions are worse on the alternate path than on the primary path, the "current" retransmission policy will not perform well. We also expect that when the conditions are better on the alternate path, performance will improve if the alternate path is used for retransmissions. However, our results show that often the latter is not true.
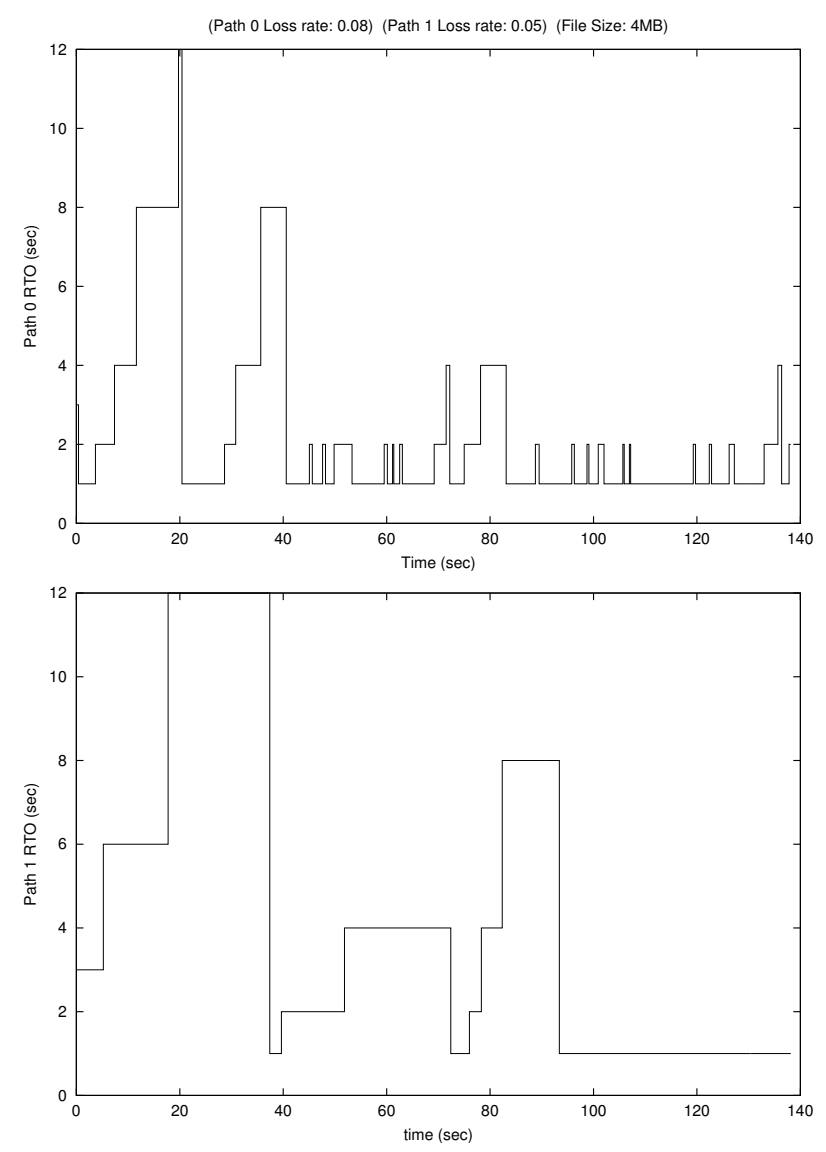

Figure F4: Example RTO dynamics of a 4MB file transfer with $8 \%$ path 0 loss rate and $5 \%$ path 1 loss rate

There are two features of SCTP which contribute to our counter-intuitive results: (1) one time only fast retransmission, and (2) Karn's algorithm. As in TCP, fast retransmissions and timeouts are the two mechanisms used in SCTP to recover from loss. Any data which has been fast retransmitted, may not be fast retransmitted again [8]. Subsequent retransmissions of the same data may only be triggered by timeouts. Hence, all data traffic on the alternate path are retransmissions, and if lost, must wait for a timeout to be retransmitted again. In and of itself, this requirement is not the problem; the same would be true if the retransmissions used the same path as the original transmissions. Due to Karn's algorithm, successful retransmissions on the alternate path cannot be used to update the round-trip time (RTT) estimation for the alternate path. Timeouts on retransmissions, however, are used to expo- nentially increase the retransmission timeout (RTO). The only traffic on the alternate path which can update the RTT estimate are the heartbeat probes used to determine destination reachability, but these heartbeats are transmitted fairly infrequently (RFC2960 recommends every 30 seconds with a random jitter of $+/-0$ to 15 seconds). In many cases the RTO is exponentially increased more frequently than can be reduced by an RTT estimate. The result is an inappropriately large RTO on the alternate path for the majority of the association.

Figure F4 illustrates the dynamics of the RTOs for path 0 (8\% loss rate) and path 1 (5\% loss rate) during a $4 \mathrm{MB}$ file transfer using the "current" SCTP. This specific transfer sent a total of 2,889 original transmissions on path 0 , of which 229 had to be retransmitted on path 1 . Of those retransmissions, 14 were retransmitted again on path $0 .^{2}$ The RTO for path 0 stays low during most of the transfer, because any successful original transmission on path 0 updates the RTT estimation and reduces the RTO (most likely back to 1 second). The average RTO for path 0 is 2.3 seconds, while path 1 has an average RTO of 5.9 seconds. In only three occasions does the RTO for path 1 get reduced, which means that during the entire transfer only three heartbeats were successfully acked. On the other hand, the graph shows seven timeouts exponentially increasing the RTO for path 1 .

\section{SOLUTIONS}

The fact that the "current" SCTP retransmission policy performs better at all (see path 1 loss rates less than 5\% in Figure F3) is evidence that retransmitting on an alternate path does have some benefits. At first, a dynamic mechanism seems ideal. The sender could use the "current" retransmission policy until the network conditions reach the threshold where the "proposed" policy performs better. Then, the sender could switch to use the "proposed" scheme. However, this solution is flawed. First, the sender cannot make an accurate estimate of the alternate paths' loss rates since they are infrequently used for retransmissions only. Second, if the threshold is met and the "proposed" scheme is used, the sender is left without a mechanism for measuring the loss rates on the alternate paths. Therefore, such a dynamic mechanism is not a valid solution.

Instead, we consider three possible solutions to improve the "current" SCTP's retransmission scheme. First, after a timeout on an alternate destination, send a heartbeat immediately. These extra heartbeat(s) would provide a mechanism for the sender to update the alternate destination(s)' RTT estimate

\footnotetext{
${ }^{2}$ The network only lost 222 SCTP packets on path 0 and 13 SCTP packets on path 1 . The sender spuriously retransmitted the others.
} 
more frequently. The drawback to this solution is that the sender would still have have few samples to estimate the alternate destination(s)' RTT.

Another possible solution is to disambiguate original transmissions from retransmissions as the Eifel algorithm does for TCP [7]. The idea is to introduce disambiguating information, such as timestamps, into the packets. Without the retransmission ambiguity problem, Karn's algorithm can be eliminated. Thus, successful retransmissions on the alternate path can be used to update the RTT estimate and keep the RTO value more accurate. This solution provides more samples to alternate destination(s) for updating their RTT estimate, but the downside is that it introduces additional overhead in each packet.

We also consider a solution which borrows the idea of a recovery period from TCP NewReno. SCTP can use such a recovery period to allow back-to-back fast retransmits; thus, reducing the number of timeouts. The recovery period would be delineated by the highest TSN (Transmission Sequence Number) outstanding at the time of a fast retransmit. Therefore, only selective acks which newly acknowledge TSNs beyond the recovery period's range would count as missing reports towards yet another fast retransmit. We believe that this mechanism may help alleviate many of the timeouts which contribute to the problem with the "current" SCTP retransmission policy.

\section{CONCLUSION AND FUTURE WORK}

The SCTP authors intentionally included a retransmission policy which fully utilizes the network redundancy available on multihomed hosts. The intended benefits of the retransmission scheme assume that loss indicates either that the destination address used is unreachable, or its network path is congested. In FCS networks, however, wireless links introduce an additional loss factor: noisy channels. It is important to understand the effects of the current SCTP retransmission policy under such conditions.

Before the retransmission policy can be optimized for wireless networks, we need to ensure that the protocol performs well on wired networks. The results presented in this paper show that there exists a flaw in the current SCTP retransmission policy. Our analysis explains that the retransmission ambiguity problem causes the current SCTP retransmission policy to perform surprisingly worse than expected.

We propose three potential solutions which should make SCTP's current retransmission scheme perform better. Future work is to investigate which of these solutions is optimal. Since these solutions are not mutually exclusive, it may be possible that a combination of these solutions is ideal.

\section{DISCLAIMER}

The views and conclusions contained in this document are those of the authors and should not be interpreted as representing the official policies, either expressed or implied, of the Army Research Laboratory or the U. S. Government.

\section{REFERENCES}

[1] CAIDA: Packet Sizes and Sequencing, Mar 1998. http://traffic.caida.org.

[2] UC Berkeley, LBL, USC/ISI, and Xerox Parc. ns2 documentation and software, Version 2.1b8, 2001. http://www.isi.edu/nsnam/ns.

[3] R. Braden. Requirements for Internet hostscommunication layers. RFC1122, Internet Engineering Task Force (IETF), October 1989.

[4] A. Caro and J. Iyengar. ns-2 SCTP module, Version 3.2, December 2002. http://pel.cis.udel.edu.

[5] K. Claffy, G. Miller, and K. Thompson. The Nature of the Beast: Recent Traffic Measurements from an Internet Backbone. INET 1998, April 1998.

[6] W. Leland, M. Taqq, W. Willinger, and D. Wilson. On the Self-similar Nature of Ethernet Traffic. In ACM SIGCOMM 1993, San Francisco, California, 1993.

[7] R. Ludwig and R. Katz. The Eifel Algorithm: Making TCP Robust Against Spurious Retransmissions. In ACM Computer Communications Review, January 2000.

[8] R. Stewart, L. Ong, I. Arias-Rodriguez, K. Poon, P. Conrad, A. Caro, and M. Tuexen. Stream Control Transmission Protocol (SCTP) Implementer's Guide. draftietf-tsvwg-sctpimpguide-07.txt, Internet Draft (work in progress), Internet Engineering Task Force (IETF), October 2002.

[9] R. Stewart and Q. Xie. Stream Control Transmission Protocol (SCTP): A Reference Guide. Addison Wesley, New York, NY, 2001.

[10] R. Stewart, Q. Xie, K. Morneault, C. Sharp, H. Schwarzbauer, T. Taylor, I. Rytina, M. Kalla, L. Zhang, and V. Paxson. Stream Control Transmission Protocol. Proposed standard, RFC2960, Internet Engineering Task Force (IETF), October 2000. 